\title{
EchoGéo
}

11 | 2010

Madagascar

\section{Acquisitions de la Bibliothèque de l'Institut de Géographie}

\section{Bernadette Joseph}

\section{OpenEdition}

Journals

Édition électronique

URL : https://journals.openedition.org/echogeo/11679

DOI : 10.4000/echogeo.11679

ISSN : 1963-1197

\section{Éditeur}

Pôle de recherche pour l'organisation et la diffusion de l'information géographique (CNRS UMR 8586)

Référence électronique

Bernadette Joseph, «Acquisitions de la Bibliothèque de l'Institut de Géographie », EchoGéo [En ligne], 11 | 2010, mis en ligne le 24 février 2010, consulté le 31 juillet 2021. URL : http:// journals.openedition.org/echogeo/11679; DOI : https://doi.org/10.4000/echogeo.11679

Ce document a été généré automatiquement le 31 juillet 2021.

EchoGéo est mis à disposition selon les termes de la licence Creative Commons Attribution - Pas d'Utilisation Commerciale - Pas de Modification 4.0 International (CC BY-NC-ND) 


\title{
Acquisitions de la Bibliothèque de l'Institut de Géographie
}

\author{
Bernadette Joseph
}

\section{NOTE DE L'ÉDITEUR}

Certains titres présents dans cette liste ne correspondent pas obligatoirement à une nouvelle acquisition du titre mais à l'acquisition d'exemplaires supplémentaires.

\section{Afrique}

1 BLANCHON, David, L'espace hydraulique sud-africain: le partage des eaux, Paris, Karthala, 2009, G 19-3485

2 Climate change and agriculture in Africa, impact assessment and adaptation strategies, London, Sterling (Va.), Earthscan, 2008, G 19-3490 DAHOU, Libéralisation et politique agricole au Sénégal, Paris, Dakar Etoile, Grand Yoff, Karthala, Crepos, Enda Graf Diapol, 2009, G 19-3494 DENANTES TEULADE, BRUNEL, Malabo, Guinée-Équatoriale : le nouvel eldorado pétrolier de l'Afrique, Paris, L'Harmattan, 2009, G 19-3505

5 FOSTER, Washed with sun : landscape and the making of white South Africa, Pittsburgh (Pa.), University of Pittsburgh Press, 2008, G 19-3493 GOLAZ, Valérie, Pression démographique et changement social au Kenya : vivre en pays gusii à la fin du XXe siècle, Paris, Nairobi, Karthala ; IFRA, 2009, G 19-3484

7 HOMOLLE, Patrick, D'une rive à l'autre: associations villageoises et développement dans la région de Kayes au Mali, Paris, L'Harmattan, 2009, G 19-3487

8 HUGON, Philippe, Géopolitique de l'Afrique, Paris, Éd. Sedes, 2009, G 19-3488

9 KASEKENDE, NDIKUMANA, Rapport sur le développement en Afrique 2008-2009 : règlement des conflits, paix et reconstruction en Afrique, Paris, Economica, 2009, G 19-3486 


\section{portuaire dans l'ouest de l'Océan Indien : essai de géographie portuaire, La Réunion, IOCL-GGA} Études, 2009, G 19-3506

LESOURNE, Gouvernance européenne et géopolitique de l'énergie: unfinished business, Paris, Bruxelles, IFRI ; Eur-Ifri, 2009, G 19-3491

MOUNDOUNGA MOUITY, Le Gabon et le nouveau partenariat africain au développement: pour une sociologie politique des institutions en Afrique, Paris, Publibook, 2009, G 19-3498

PICAZO, ZHAO,Zambia health sector public expenditure review : accounting for resources to improve effective service coverage, Washington, The World Bank, 2009, G 19-3503

POURTIER, Roland, Atlas de l'UDEAC, Paris, Ministère de la coopération, 1993, G 19-3489

SMITH, LEVASSEUR, Atlas de l'Afrique; supplément La percée chinoise en Afrique, Paris, Autrement, 2009, G 19-3501

SY, Géographie urbaine de l'insalubrité : le cas de Saint-Louis du Sénégal, Paris, L'Harmattan, 2009, G 19-3495

TICHIT, Christine, Understanding the family composition of households in demographic and health surveys, Paris, CEPED, 2008, G 19-3492

VALLETTE, CAUSSE, LENGAGNE ... Madagascar, l'île océan, Paris, Boulogne-sur-Mer, Perpignan, Autrement, Nausicaá, Mare nostrum, 2009, G 19-3497

WALTHER, RETAILLÉ, Affaires de patrons : villes et commerce transfrontalier au Sahel, Bern, P. Lang, 2008, G 19-3496

ZHOU,Decentralization, democracy, and development : recent experience from Sierra Leone, Washington, The World Bank, 2009, G 19-3502

\section{Amérique du nord}

DRACHE, GERTLER, The New era of global competition : state policy and market power, Montreal, Buffalo, McGill Queen's University Press, 1991, G 20-1637

2 LE GOIX, SUSS, DANIEL, Atlas New York, Paris, Autrement, 2009, G 20-1638

SAINT-PIERRE, Adéodat, Défendre la ruralité: témoignage d'un agriculteur, Rimouski, GRIDEQ, 2007, G 20-1634

4 SAVOIE, RAYNAULD, Essais sur le développement régional, Montréal, Les Presses de l'Université de Montréal, 1986, G 20-1639

\section{Amérique latine}

Argentine youth : an untapped potential, Washington, The World Bank, 2009, G 21-1704

AZEVEDO, COURLET, ARAUJO DE MORAES FIL, Territoire et développement économique au Brésil: les arrangements productifs locaux au Pernambuco, Paris, L'Harmattan, 2009, G 21-1707

CABANES, GEORGES, São Paulo : la ville d'en bas, Paris, L'Harmattan, 2009, G 21-1702

DABÈNE, BOISSIÈRE, Atlas de l'Amérique latine: les révolutions en cours, Paris, Éd. Autrement, 2009, G 21-1696 
GABBI, Ligia Villas Boas, Gouverner une mégapole: l'expérience de Sao Paulo, Paris, l'Harmattan, 2009, G 21-1695

GUIBERT, CARRIZO, LIGRONE, Le bassin du Rio de la Plata : développement local et intégration régionale, Toulouse, Presses universitaires du Mirail, 2009, G 21-1701

GUILLEN, Tradições \& traduções: a cultura imaterial em Pernambuco, Recife, Ed. universitária UFPE, 2008, G 21-1698

HARTOG, DESSE, Les littoraux de la Caraibe: pratiques sociales et nouvelles dynamiques spatiales, Paris, Éd. Karthala, 2009, G 21-1697

Increasing formality and productivity of Bolivian firms, Washington, The World Bank, 2009, G 21-1703

JALET, L' urgence, l'échéance, la durée, Paris, Archibooks-Sautereau, 2008, G 21-1706

LAFFITE, Christiane, Histoire des côtes colombiennes : navigation, commerce et guerres civiles à l'époque de Bolivar, Paris, L'Harmattan, 2003, G 21-1699

TSIKATA, MOREIRA, HAMILTON,Accelerating trade and integration in the Caribbean : policy options for sustained growth, job creation, and poverty reduction, Washington, The World Bank, 2009, G 21-1705

37 VIANÈS, Guyane, la ruée vers l'or : mémoire, Mouriès, Terriciaë éd., 2008, G 21-1700

\section{Asie}

BROWN,The territories of Indonesia, London, New York, Routledge, 2009, G 17-2717

CABRILLAC, Économie de la Chine, Paris, Presses universitaires de France, 2009, K 1074

CONNELL, WADDELL, Environment, development and change in rural Asia-Pacific : between local and global, London, New York, Routledge, 2007, G 17-2719

COSAERT, Les enjeux du Pacifique, Paris, Ellipses, 2009, G 17-2711

DORRONSORO, Que veut la Turquie ?: ambitions et stratégies internationales, Paris, Autrement, 2009, G 17-2718

DOVERT, TRÉGLODÉ, Viêt Nam contemporain, Paris, Bangkok, Les Indes savantes ; Institut de recherche sur l'Asie du sud est contemporaine, 2009, G 17-2714

KONINCK, Rodolphe de, L'Asie du Sud-Est, Paris, A. Colin, 2009, G 17-2712

LEVEAU, L'Asie du Sud-Est 2009 : les évenements majeurs de l'année, Bangkok, Paris, IRASEC ; Lignes de repères, 2009, G 17-2715

PENSEREAU, Michel, Le Japon entre ouverture et repli à travers l'histoire : un rapport cyclique au monde, Paris, l'Harmattan, 2009, G 17-2713

SANJUAN, BENOÎT-GUYOD, DANIEL, Atlas Shanghai, Paris, Autrement, 2009, G 17-2716

\section{Atlas}

Atlas de México y del mundo, México, Madrid, Nuevas tecnicas educativas, Santillana, 1978, AQ 21-45

Atlas Four, Glasgow, Harlow, Collins, Longman, 1973, AQ 9-68 

Provence, Aubagne, Etang de Berre, Marseille, INSEE PACA, DDE 13, AGAM, 2002, AQ 13-129

\section{Bibliographie} et manuscrits relatifs à l'Afrique occidentale existant au Gouvernement général de l'A.O.F. à Dakar, Laval, Goupil, 1926, G 1-831

Atlas three, Glasgow, Harlow, Collins, Longman, 1973, AQ 9-58

BEN YAHMED, HOUSTIN, PITTE, Atlas de l'Afrique, Paris, Ed. du Jaguar, 2009, G 19-3504

BONAPACE, MOTTA, Atlante della produzione e dei commerci, Novara, Istituto geografico de Agostini, 1967, AQ 9-72

BONAPACE, MOTTA, Atlante geografico metodico, Novara, Istituto geografico de Agostini, 1969, AQ 9-62

CHARLIER, Atlas du 21e siècle, Paris, Nathan, 2009, AQ 9-56

DAVID, Atlas mondial, Paris, Auzou, 2009, AQ 9-61

EFIMOV, Atlas geografičeskih otkrytij v Sibiri $i$ v Severo-Zapadnoj Ameriki XVII-XVIII vv., Moskva, Nauka, 1964, AF 17-72

EGGERS, PFROMMER,Die Länder der Erde : Harms Atlas, München, P. List Verl., Kartographische Anstalt, 196X, AQ 9-64

FLORENT, Atlas des pays du monde, Paris, Larousse, 2009, A 9.0160

FULLARD,Philips' modern Commonwealth atlas, London, G. Philip, 1967, AQ 9-70

FULLARD,Philips' record atlas, London, G. Philip, E. Stanford, 1961, AQ 9-66

FULLARD, The Whitehall atlas, London, E. Stanford, 1960, AQ 9-67

Hac'kacan sovetakan soc'ialistakan respowblikac'i Atlas [Atlas de l'Arménie], Erevan, Moskva, 1961, AQ 17-80

Hammond's library world atlas, New York, Maplewood, C.S. Hammond, 1952, AQ 9-65

HAWES, KNIGHT,Atlas of man and religion, Oxford, The religious educational press, 1970, AQ 9-69

MÉRIENNE, Atlas mondial, Rennes, Éd. Ouest-France, 2009, A 9.0 163

ORMELING, De Grote Bosatlas, Gronigen, Wolters-Noordhoff, 1971, AQ 9-63

PINOL, GARDEN, Atlas des Parisiens : de la Révolution à nos jours: population, territoire et habitat, productions et services, religion, culture, loisirs, Paris, Parigramme, 2009, AQ 13-127

El uso del suelo en la República Mexicana: atlas, México, Secretaría de Agricultura y Recursos Hidraúlicos, Subsecretaría de Agricultura y Operación, Dirección General de Agricultura, 1979, AF 21-40

SIMÉONI, Patricia, Atlas du Vanouatou (Vanuatu), Port-Vila, Éd. Géo-consulte, 2009, AQ 17-79

Références générales de Gabriel Wackermann, ... Strasbourg, 2009, G 1-833 


\section{Congrès}

BAIL, Santé et mondialisation: quels impacts pour la France ?, Montrouge, J. Libbey Eurotext, 2008, G 3-3090

BERRAMDANE, ROSSETTO, La politique européenne d'immigration, Paris, Karthala, 2009, G 3-3112

BOCHER, ERTZ, OGRS 2009: abstract proceedings, Paris, Nantes, Publibook, Éd. Centrale Nantes, 2009, G 3-3117

BOISVERT, HAMEL, Redéploiement industriel et planification régionale, Montréal, Université de Montréal, Faculté de l'aménagement, 1985, G 3-3106

BRISEBARRE, FABRE, LEBAUDY, Sciences sociales, regards sur le pastoralisme contemporain en France[actes de la rencontre nationale, 13 novembre 2008, Montpellier], Die, Saint-Martinde-Crau, Laudun, Association française de pastoralisme, Maison de la transhumance, Cardère éd., 2009, G 3-3111

Cartographie et planification au Tchad: colloque de N'Djamena 14 et 15 janvier 1977: atlas régionaux et régionalisation $\mathrm{du}$ plan au Tchad: régionalisation, urbanisation, développement rural, nomadisme et pastoralisme, milieu physique, télédétection, N'Djamena ; Paris, [s.n.], 1977, G 3-3092

CELIMENE, GRANVORKA, Confrontation et collision du réel et de l'imaginaire de six ports francophones: Bordeaux (France), Saint Louis (Sénégal), Fort-de-France (Martinique), Jacmel (Haïti), Shédiac (Nouveau Brunswick), Québec (Canada), Paris, Publibook, 2008, G 3-3110

CHARBONNEAU, LEWIS, MANZAGOL, Villes moyennes et mondialisation: renouvellement de l'analyse et des stratégies: [colloque, Montréal, du 18 au 20 septembre 2002], Montréal, Ed. Trames, 2003, G 3-3108

DIALLO, Les États-nations face à l'intégration régionale en Afrique de l'Ouest: le cas de la république de Guinée, Paris, Éd. Karthala, 2009, G 3-3104

DURAND, JACQUET, Centre et périphérie: approches nouvelles des orientalistes: actes du colloque, les 31 mai et 1er juin 2006, Paris, Collège de France, Paris, J. Maisonneuve, 2009, G 3-3100

DUREAU, HILY, RIEFFEL, Les mondes de la mobilité, Rennes, Presses universitaires de Rennes, 2009, G 3-3115

GOEH-AKUE, Les États-nations face à l'intégration régionale en Afrique de l'Ouest: le cas du Togo, Paris, Éd. Karthala, 2009, G 3-3102

HAU, Regards sur le capitalisme rhénan, Strasbourg, Presses universitaires de Strasbourg, 2009, G 3-3098

HUISSOUD, MUNIER, La guerre économique: rapport Anteios 2010, Paris, Presses universitaires de France, 2009, G 3-3089

L'identité européenne: ses fondements historiques et géographiques, Paris, Société de géographie, 2009, G 3-3094

8 LAGADEC, LE BIHAN, TANGUY, Le canton un territoire du quotidien ?, Actes du colloque organisé à l'Université Rennes 2 Haute-Bretagne, 21-23 septembre 2006, Rennes, Presses universitaires de Rennes, 2009, G 3-3093

MERLE, L'estuaire de la Seine : un passé en commun, un avenir en construction, Rouen, Éd. des Falaises, 2009, G 3-3099 

2009, G 3-3101

\section{Dictionnaires} 395

REYNARD, CORATZA, REGOLINI-BISSIG, Geomorphosites, München, Verl. Dr. F. Pfeil,

Tensions foncières sur le littoral, Rennes, Presses universitaires de Rennes, 2009, G 3-3096

TREMBLAY, TREMBLAY, La compétitivité urbaine à l'ère de la nouvelle économie : enjeux et défis, Sainte-Foy, Presses de l'Université du Québec, 2006, G 3-3107

Urbanisme raisonné en milieu rural: actes des journées d'études 1er, 2 octobre 2008, Agen : de nouveaux modes d'habiter à inventer, CAUE 47, 2009, G 3-3118

URSS et Russie : actes 2008, Bordeaux, SCÉRÉN-CRDP Aquitaine, 2009, G 3-3116

WILEY, Estuarine processes, New York, Academic Press, 1976, G 3-3091

YÉO, Les États-nations face à l'intégration régionale en Afrique de l'Ouest: le cas de la Côte d'Ivoire, Paris, Éd. Karthala, 2009, G 3-3103

ALARY, BAZILE, BERLAND, Nourrir les hommes : un dictionnaire, Neuilly, Atlande, 2009, W

ANTONI, Lexique de la ville, Paris, Ellipses, 2009, W 400

BIORET, Frédéric, Dictionnaire de la protection de la nature, Rennes, Presses Universitaires de Rennes, 2009, W 385

DEUBEL, MONTOUSSÉ, Dictionnaire des auteurs en sciences économiques et sociales, Rosnysous-Bois, Bréal, 2009, W 390

FERRÉOL, Dictionnaire de l'Union européenne, Paris, A. Colin, 2000, W 392

GEORGE, VERGER, Dictionnaire de la géographie, Paris, PUF, 2009, W 388

GERBET, BOSSUAT, GROSBOIS, BARNAVI, Dictionnaire historique de l'Europe unie, Bruxelles, A. Versaille, 2009, W 387

GIBLIN-DELVALLET, Dictionnaire des banlieues, Paris, Larousse, 2009, W 386

GREFFE, LALLEMENT, DE VROEY, Dictionnaire des grandes oeuvres économiques, Paris, Dalloz, 2002, W 391

JOUTY, ODIER, Dictionnaire de la montagne, Paris, Omnibus, 2009, W 393

Le petit Larousse illustré 2010 en couleurs : 87000 articles, 5000 illustrations, 321 cartes, chronologie universelle, Paris, Larousse, 2009, V 104

VAÏSSE, MORELLE, Dictionnaire des relations internationales de 1900 à nos jours, Paris, A. Colin, 2009, W 394

Vocabulaire thématique de géographie: français-anglais, anglais-français: le vocabulaire d'indexation sélectionné et utilisé par l'équipe de rédaction de la Bibliographie géographique internationale, Paris, CNRS-Pôle de recherche pour l'organisation et la diffusion de l'information géographique, 2009, W 396

10 Vocabulaire thématique de géographie: français-espagnol, espagnol-français: le vocabulaire d'indexation sélectionné et utilisé par l'équipe de rédaction de la Bibliographie géographique internationale, Paris, CNRS-Pôle de recherche pour l'organisation et la diffusion de l'information géographique, 2009, W 398 


\section{Europe}

11 ALBERTOS PUEBLA, Transporte, movilidad y sostenibilidad, Valencia, Universitat de Valencia, Facultat de geografia i història, 2008, G 15-5997

BAILONI, PAPIN, DUMAS, Atlas géopolitique du Royaume-Uni : les nouveaux défis d'une vieille puissance, Paris, Autrement, 2009, G 15-5999

BARNAVI, Élie, L'Europe frigide : réflexions sur un projet inachevé, Bruxelles, A. Versaille, 2008, G 15-5953

BARON-YELLÈS, BOISSIÈRE, Atlas de l'Espagne: une métamorphose inachevée, Paris, Autrement, 2009, G 15-5977

BAVOUX, Jean-Jacques, La géographie: objet, méthodes, débats, Paris, A. Colin, 2009, G 15-1782

BÉNARD, Nicolas, Les périphéries du Norden, Paris, Choiseul-éditions, 2008, G 15-5963

BERGER, CHEVALIER, CORTES, Héritages et trajectoires rurales en Europe, Paris, L'Harmattan, 2009, G 15-5958

BERGERET-CASSAGNE, Pour une Europe fédérale des collectivités locales: un demi-siècle de militantisme du Conseil des communes et des régions d'Europe, 1950-1999, Paris, l'Harmattan, 2009, G 15-5991 BOVET, Philippe, Écoquartiers en Europe, Mens, Terre vivante, 2009, G 15-5949

BRUCKMEIER, TOVEY, Rural sustainable development in the knowledge society, Farnham, Surrey, England, Burlington, (VT), Ashgate, 2009, G 15-5966

Le Caucase : un espace de convoitise, Paris, La Documentation française, 2009, G 15-6005

, STRICKNER, TROUVÉ, Souveraineté alimentaire: que fait l'Europe?: pour une nouvelle politique agricole et alimentaire européenne, Paris, Syllepse, 2009, G 15-5938

CLOCHARD, Atlas des migrants en Europe: géographie critique des politiques migratoires européennes, Paris, A. Colin, 2009, G 15-5972

DECOSTER, VERSINI, TAJANI ... UE : la politique des transports : vers une mobilité durable, Paris, la Documentation française, 2009, G 15-6001

DEHOUSSE, DELOCHE-GAUDEZ, JACQUOT, Que fait l'Europe ? Paris, Presses de Sciences Po, 2009, G 15-5942

DiNH, Les métropoles: nouveaux défis des villes européennes, Paris, Autrement, 2009, G 15-5989

DUPEYRON, MORATA, L'Europe au défi de ses régions transfrontalières : expériences rhénane et pyrénéenne, Berne, P. Lang, 2008, G 15-6000

EJDERYAN, Une renaturation en béton !: comprendre la participation et la nature dans les renaturations de cours d'eau suisses au regard d'une théorie de la pratique, Zürich, Geographisches Institut, Abteilung Humangeographie, 2009, G 15-6007

ESSAÏAN, Moscou: portrait de ville [numéro spécial d'Archiscopie], Paris, Cité de l'architecture et du patrimoine ; Institut français d'architecture, 2009, G 15-5955

L'Espagne, les métamorphoses d'une puissance européenne, Paris, Association des professeurs d'histoire et de géographie de l'enseignement public, 2009, G 15-6003

131 FERRÉOL, GONTHIER, Universités et territoires : une comparaison européenne, Paris, SUDEL, 2008, G 15-5986 

2009, G 15-5965 15-5976 2009, G 15-5959 15-5982 15-6006 G 15-6004 G 15-5975 2009, G 15-5990 2009, G 15-5951 15-5956

FERRERA, Maurizio, Les nouvelles frontières du social: l'intégration européenne et les transformations de l'espace politique de la protection sociale, Paris, Presses de Sciences Po,

FOUCHER, L'Europe : entre géopolitiques et géographies, Paris, Sedes, Cned, 2009, G 15-5994

FOURMONT-DAINVILLE, CHERKAOUI, Madrid : régénérations, Paris, Autrement, 2009, G

FRÉVILLE, La mobilité des jeunes, Marly-le-Roi, Institut national de la jeunesse, des sports et de la vie associative, Centre de documentation, 2008, G 15-5980

FUSARO, Les Italo-argentins en Italie (1998-2006) : "retour aux racines" ou nouveau départ? : paradoxes d'un mouvement migratoire contemporain, Torino, Paris, L'Harmattan Italia, L'Harmattan, 2009, G 15-5987

GOUGEON, L'Allemagne, une nouvelle puissance?, Paris, IRIS ; Dalloz, 2009, G 15-5950

GRÉSILLON, Boris, L'Allemagne, vingt ans après..., Paris, la Documentation française ; IESR,

GUILLERME, Bâtir la ville: révolutions industrielles dans les matériaux de construction: France-Grande-Bretagne (1760-1840), Seyssel, Champ vallon, 1995, G 15-5992

INDOVINA, La ciudad de baja densidad : lógicas, gestión y contención, Barcelona, Diputació Barcelona, 2007, G 15-5969

JEAN, BAUDELLE, GUELLEC, L'Europe : aménager les territoires, Paris, A. Colin, 2009, G

Jeunes en errance: les conditions d'un retour: étude de faisabilité sur la réintégration des mineurs isolés victimes de traite, Espagne, France, Italie, Albanie, Autriche, Roumanie, Paris, Forum urbain pour la sécurité urbaine, 2009, G 15-5964

KRIEG-PLANQUE, Purification ethnique : une formule et son histoire, Paris, CNRS éd., 2003, G

LABOREY, BALTROCK, Berlin : quoi de neuf depuis la chute du Mur ?,Paris, Autrement, 2009,

LABRIANIDIS, The moving frontier : the changing geography of production in labour-intensive industries, Aldershot, Burlington (Vt.), Ashgate, 2008, G 15-5967

LETTIERI, Comprendre l'Italie des années 2000: du social au politique, Aix-en-Provence, Publications de l'Université de Provence, 2009, G 15-5943

MACCAGLIA, Palerme : illégalismes et gouvernement urbain d'exception, Lyon, ENS éd., 2009,

MAGNETTE, Le régime politique de l'Union européenne, Paris, Les Presses de Sciences Po,

De nouvelles mobilités dans une Europe élargie, Villeneuve d'Ascq, Université des sciences et technologies de Lille, 2008, G 15-5998

REYNIÉ, L'opinion européenne en 2009, Paris, Lignes de repères ; Fondation R. Schuman,

SÁNCHEZ SÁNCHEZ, José, Geografía de Albacete: factores del desarrollo económico de la provincia y su evolución reciente, Albacete, Instituto de estudios albacetenses, 1982, G 


\section{France} 2009, G 10-5840 10-5788

Sandstone landscapes, Praha, Academia, Bohemian Switzerland, National Park Administration, Royal Botanic Gardens Kew, 2007, G 15-5978

SPECHT, Judith, Fernwandern und Pilgern in Europa : über die Renaissance der Reise zu Fuss, München, Profil Verl., 2009, G 15-5968

SUÁREZ DE VIVERO, RODRÍGUEZ MATEOS, Atlas de la Europa marítima : jurisdicciones, usos y gestión, Barcelona, Serbal, 2007, G 15-6008

VAZ, NIJKAMP, RASTOIN, Traditional food production and rural sustainable development : a european challenge, Farnham (England), Burlington (VT), Ashgate, 2009, G 15-5981

VERHOFSTADT, Guy, Sortir de la crise: comment l'Europe peut sauver le monde, Arles, Bruxelles, Actes Sud ; A. Versaille, 2009, G 15-5952

VIDAL, Patrice, Dans l'arrière-cour de Moscou, Paris, F.-X. de Guibert, 2009, G 15-5940

WACKERMANN, L'Europe : approche géographique, Paris, Ellipses, 2009, G 15-5979

WEBER, Serge, Nouvelle Europe, nouvelles migrations : frontières, intégration, mondialisation, Paris, Le Félin, 2007, G 15-5947

ADENOT, Penser et faire l'urbanisme de Clermont-Ferrand, 1998-2008, Nohanent, Éd. Revoir,

BIGOTEAU, GARAT, MOREAU, Les jeunes dans la ville: atlas social de Nantes Métropole, Rennes, Presses universitaires de Rennes, 2009, G 10-5830

BOCHEUX, Antoine, Histoire de l'île Nouvelle : naissance et évolution d'une île de l'estuaire de la Gironde, Bordeaux, Association Océan, 2008, G 10-5805

BONNIER, Gaston, Les noms des fleurs trouvés par la méthode simple sans aucune notion de botanique, Paris, Librairie générale de l'enseignement, 1960, G 10-5802

BOURGAU, TREYER, La forêt française en 2050-2100 : essai de prospective, Besançon, Conseil général de l'agriculture, de l'alimentation et des espaces ruraux; Cêtre, 2009, G

BOYER, Jean-Claude, La France : les 26 régions, Paris, A. Colin, 2009, G 10-5795

Changement climatique, énergie et développement durable des territoires, Paris, La Documentation française, 2005, G 10-5817

Les chiffres clés du tourisme de montagne en France, Paris, Atout France, 2009, G 10-5836

COUREL, Des paysages à voir et à lire en Bourgogne, Dijon, Éducagri ; Centre de culture scientifique technique et industrielle de Bourgogne, 2009, G 10-5784

DARTOUT, CORDOBES, Une nouvelle ambition pour l'aménagement du territoire, Paris, la Documentation française, 2009, G 10-5833

DELFANTE, La Part-Dieu : le succès d'un échec, Lyon, Libel, 2009, G 10-5843

DESPRÉS, L' estuaire de la Loire: un territoire en développement durable?, Rennes, Presses universitaires de Rennes, 2009, G 10-5841

DRACIUS-PINALIE, SAM, LONG ... La crise de l'outre-mer français: Guadeloupe, Martinique, Réunion, Paris, L'Harmattan, 2009, G 10-5829 
FLONNEAU, GUIGUENO, LATOUR, De l'histoire des transports à l'histoire de la mobilité ? : état des lieux, enjeux et perspectives de recherche, Rennes, Presses universitaires de Rennes, 2009, G 10-5835

Le fonctionnement métropolitain du Bassin parisien : du Bassin parisien à la région économique de Paris, 2004-2008, Paris, DIACT, 2009, G 10-5808

FRÉMONT, Normandie sensible, Paris, Éd. Cercle d'Art, 2009, G 10-5824

FRÉMONT, La mémoire d'un port : Le Havre, Paris, Arléa, 2009, G 10-5825

FRÉMONT-VANACORE, Anne, La France en Europe, Paris, A. Colin, 2009, G 10-5798

GAY, Les cocotiers de la France : tourismes en outre-mer, Paris, Belin, 2009, G 10-5834

GEIPEL, FINN, Grand Paris métropole douce : hypothèses sur le paysage post-Kyoto, Paris, J. M. Place ; diff. Beauchesne, 2009, G 10-5797

GILLI, Paris, une métropole dans le monde : état des lieux et mise en perspective des savoirs, Paris, DIACT, 2007, G 10-5807

GLINER, Lyon, Grenoble : la nécessité d'une mégalopole, [S.l.][s.n.], 2009, G 10-5822

HAMMAN, BLANC, HENNINGER ... Sociologie du développement durable urbain : projets et stratégies métropolitaines françaises, Bruxelles, PIE P. Lang, 2009, G 10-5826

HELLIER, La France, la ressource en eau: usages, gestions et enjeux territoriaux, Paris, A. Colin, 2009, G 10-5827

HINNEWINKEL, LAVAUD, Vignobles et vins en Aquitaine: images et identités d'hier et d'aujourd'hui, Pessac, Maison des sciences de l'homme d'Aquitaine, 2009, G 10-5814

IOGNA-PRAT, ZADORA-RIO, La paroisse: genèse d'une forme territoriale, Saint-Denis, Presses universitaires de Vincennes, 2006, G 10-5815

Le golfe du Lion: un observatoire de l'environnement en Méditerranée, Versailles, Éditions Quæ, 2009, G 10-5783

JALABERT, Guy, Mémoires de Toulouse, ville d'hier, ville d'aujourd'hui, Toulouse, Presses universitaires du Mirail, 2009, G 10-5787

JEAN, PÉRIGORD, Géographie rurale : la ruralité en France, Paris, A. Colin, 2009, G 10-5812

JEAN, VANIER, GUIGOU, La France: aménager les territoires, Paris, A. Colin, 2009, G 10-5800

JULIEN-LABRUYÈRE, HELBERT, Agricultures et paysages: témoignages et points de vue des CAUE, Dijon, Paris, Educagri ; FNCAUE, 2009, G 10-5804

LAU, L'état de la France 2009-2010: un panorama unique et complet de la France, Paris, La Découverte, 2009, G 10-5794

LIGNON - DARMAILLAC, L'œnotourisme en France: nouvelle valorisation des vignobles: analyse et bilan, Bordeaux, Féret, 2009, G 10-5813

MANTEI, MAINBOURG, REMOND, Tourisme durable dans les îles: protéger, développer, valoriser, Paris, ODIT France, 2009, G 10-5803

MAURIN, CLERC, Déchiffrer la société française, Paris, la Découverte, 2009, G 10-5828

NOIN, Daniel, Le nouvel espace français, Paris, A. Colin, 2009, G 10-5785

NOUVEL, DUTHILLEUL, CANTAL-DUPART, Naissances \& renaissances de mille et un bonheurs parisiens, Paris, les Éd. du Mont-Boron, 2009, G 10-5837 
197 L' offre d'enseignement supérieur et de recherche face aux besoins de l'économie et de l'emploi dans les territoires du Bassin parisien: 2006-2008, Paris, Délégation interministérielle à l'aménagement et à la compétitivité des territoires, 2009, G 10-5806

$L^{\prime}$ offre de services en milieu rural: quels outils?, Paris, ETD, Entreprises territoires \& développement, 2008, G 10-5831

PAVIE, KALFON, Tourisme et innovation : comment décrypter les tendances qui bouleversent le monde du voyage?, Paris, L'Harmattan, 2009, G 10-5810

PIERCY, La France : le fait régional, Paris, Hachette supérieur, 2009, G 10-5821

PITTE, Jean-Robert, La France, Paris, A. Colin, 2009, G 10-5789

Pôles et métropoles, Paris, La Documentation française, 2005, G 10-5816

La politique parisienne pour le logement: bilan 2001 à 2006, perspectives 2007 et 2008: communication au Conseil de Paris sur l'effort de la collectivité parisienne en matière de logement : bleu budgétaire Logement, Paris, Mairie de Paris, 2007, G 10-5809

Prospective des territoires, Paris, La Documentation française, 2006, G 10-5818

Schéma directeur de la région île-de-France: le résumé: projet adopté par délibération du Conseil régional le 25 septembre 2008, Paris, Conseil régional d'Île-de-France, G 10-5832

Seine Métropole: Paris Rouen Le Havre : le diagnostic prospectif de l'agglomération parisienne, Paris, Archibooks-Sautereau, 2009, G 10-5838

VACHER, Antoine, Fleuves et rivières de France: études sur les documents réunis par l'administration des ponts et chaussées, Paris, Impr. Nationale, 1908, G 10-5801

VERGER, GHIRARDI, Zones humides du littoral français : estuaires, deltas, marais et lagunes, Paris, Belin, 2009, G 10-5823

\section{Généralités}

BÉGUIN, CERF, Dynamique des savoirs, dynamique des changements, Toulouse, Octares, 2009, G 5-1790

BRIGAND, Louis, Besoin d'îles, Paris, Stock, 2009, G 5-1780

CAPO-CHICHI, DURAND, Guide juridique de l'image, Paris, Pyramyd, 2009, G 5-1789

CLOUT, Jacqueline Beaujeu-Garnier, 1917-1995, London, Continuum, 2009, BGEN 1011

CLOUT,Patronage and the production of geographical knowledge in France : the testimony of the first hundred regional monographs, 1905-1966, London, Historical geography research group, 2009, G 5-1791

4 CLOUT, GAY, De la géographie à l'action: Jacques Levainville, militaire géographe et homme d'affaires, Rouen, Études normandes, 2004, BGEN 1012

CUADRAT, MARTÍN VIDE, La climatología española: pasado, presente y futuro, Zaragoza, Prensas universitarias de Zaragoza, 2007, G 5-1792

DUPUY, En relisant Jules Verne: un autre regard sur Les voyages extraordinaires, Dôle, La clef d'argent, 2005, G 5-1787

DUPUY, Jules Verne, l'homme et la Terre: la mystérieuse géographie des Voyages extraordinaires, Dôle, La clef d'argent, 2006, G 5-1788

FARINELLI, BIENVENU, GRUET, De la raison cartographique, Paris, CTHS, 2009, G 5-1785 

2009, G 9-7191 2008, G 9-7089 9-7115 9-7075

GRATALOUP, L'invention des continents: comment l'Europe a découpé le monde, Paris, Larousse, 2009, G 5-1793

Histoire des sciences et des techniques, Paris, Association des professeurs d'histoire et de géographie de l'enseignement public, 2009, G 15-6002

LE ROY LADURIE, Emmanuel, Remise à Jean-Robert Pitte de son épée d'académicien: Sorbonne, salons du rectorat de Paris, le 16 octobre 2008, Paris, Fayard, 2009, G 5-1778

PELLETIER, Élisée Reclus, géographie et anarchie, Paris, Saint-Georges-d'Oléron, les Éd. du Monde libertaire, les Éd. Libertaires, 2009, G 5-1794

ROBIC, TISSIER, Philippe Pinchemel: une intelligence géographique contemporaine, Paris, Association des professeurs d'histoire et de géographie de l'enseignement public, 2009, G 15-6003-<p. 31-40>

TIFFOU, Jacky, Commenter la carte topographique aux examens et concours, Paris, A. Colin, 2009, G 5-1781

\section{Géographie humaine}

ABECASSIS, BERGEZ, Les filières céréalières: organisation et nouveaux défis, Versailles, Éditions Quae, 2009, G 9-7083

Abortion worldwide : a decade of uneven progress, New York, Alan Guttmacher Institute,

Agriculture et biodiversité: valoriser les synergies : expertise scientifique collective Inra, Juillet 2008, Versailles, Éditions Quae, 2009, G 9-7069

AGUDELO ALVARADO, BOIDIN-CARAVIAS, SANSONE, Autour de "l'Atlantique noir": une polyphonie de perspectives, Paris, IHEAL éditions, 2009, G 9-7138

L'alimentation du monde et son avenir, Paris, l'Harmattan, 2009, G 9-7068

AMADOU SANNI, KLISSOU, Marcoux ... Villes du Sud: dynamiques, diversités et enjeux démographiques et sociaux, Paris, Éditions des Archives contemporaines, Agence universitaire de la francophonie, 2009, G 9-7164

AMATI, Urban green belts in the twenty-first century, Aldershot, Burlington (Vt), Ashgate,

ARDENNE, Cityrama : FGP(u), Paris, Archibooks-Sautereau, 2008, G 9-7172

BAUDIN, BONNIN, Faire territoire, Paris, Recherches, 2009, G 9-7168

BAUDOUI, Rémi, Géopolitique du terrorisme : les territoires de l'insécurité de la mondialisation, Paris, A. Colin, 2009, G 9-7066

BIASE, CORALLI, Espaces en commun: nouvelles formes de penser et d'habiter la ville, Paris, L'Harmattan, 2009, G 9-7190

BEALL, Jo, Cities and development, Oxon, New York, Routledge, 2009, G 9-7092

BÉNASSY-QUÉRÉ, CHEVALLIER, L'économie mondiale 2010, Paris, La Découverte, 2009, G

BICKNELL, DODMAN, SATTERTHWAITE, Adapting cities to climate change : understanding and addressing the development challenges, London, Sterling (VA), Earthscan, 2009, G 
BONIFACE, VÉDRINE, MAGNIER, Atlas des crises et des conflits, Paris, A. Colin, Fayard, 2009, G 9-7136

BRETON, LE LOUARN, SAFFACHE, Patrimoine culturel et tourisme alternatif: Afrique, Amériques, Caraibe, Europe, Paris, Pointe-à-Pitre, Karthala, Créjéta, 2009, G 9-7162

BROWN, Lawrence A., Innovation diffusion : a new perspective, London, New York, Methuen, 1981, G 9-7108

CASELLI, VALLIN, WUNSCH, Démographie: analyse et synthèse, Paris, Ed. de l'Institut national d'études démographiques, 2006, G 9-5026-<8>

CHALMIN, Philippe, Du rare à l'infini: panorama mondial des déchets 2009, Paris, Economica, 2009, G 9-7065

CHEVERRY, GASCUEL-ODOUX, Sous les pavés la terre: connaitre et gérer les sols urbains, Montreuil, Omniscience, 2009, G 9-7129

CHERVEL, Mondialisation des marchandises et marchandisation du monde : matériau de pensée économique pour le nouveau siècle, Paris, Publisud, 2008, G 9-7174

COLLINS, Andrew E., Disaster and development, Oxon, New York, Routledge, 2009, G 9-7093

COLLIN, La bombe: l'univers opaque du nucléaire, Paris, Éditions Autrement, 2009, G 9-7155

CONTE, ZERBATO, La tiers-mondialisation de la planète, Pessac, Presses universitaires de Bordeaux, 2009, G 9-7151

CORTES, FARET, Les circulations transnationales: lire les turbulences migratoires contemporaines, Paris, A. Colin, 2009, G 9-7125

COUSIN, RÉAU, Sociologie du tourisme, Paris, La Découverte, 2009, G 9-7133

La crise alimentaire qui vient, Paris, Alternatives économiques, 2009, G 9-7081

Culture et développement, la durabilité renouvelée par l'approche interculturelle ?, Paris, Publibook, 2009, G 9-7169

DARIN, La comédie urbaine, Gollion (Suisse), InFolio, 2009, G 9-7163

DELORY-MOMBERGER, Christine, Le sujet dans la cité: insertion, territoires solidaires, Nantes, Éditions Pleins Feux, 2009, G 9-7072

Le développement durable comme genre de vie: dossier, Paris, Institut catholique de Paris, Desclée De Brouwer, 2009, G 9-7180

DRESSLER-HOLOHAN, Eurasie: espace mythique ou réalité en construction ?, Bruxelles, Bruylant, 2009, G 9-7167

DUHAMEL, VIOLIER, Tourisme et littoral : un enjeu du monde, Paris, Belin, 2009, G 9-7185

DUMONT, LE BRAS, Doit-on contrôler l'immigration ?, Bordeaux, Éd. Prométhée, 2009,

G 9-7148

Economie de l'alimentation, Paris, CERAS, 2008, G 9-7085

Emotion, place, and culture, Farnham, Burlington, (VT), Ashgate, 2009, G 9-7079

Enfance et développement, Bruxelles, De Boeck université, 2009, G 9-7140

EPSTEIN, JAFFRELOT, Les nouveaux géants: comprendre les pays émergents: Chine, Inde, Brésil, Russie, Paris, L'Express; Sciences Po-CERI et Atelier de cartographie, 2008, G 9-7063 
ESTIVAL, Le marché mondial des ressources : la guerre fait rage, Paris, l'Harmattan, 2009, G 9-7157

FAINSTEIN, CAMPBELL, Readings in urban theory, Cambridge (Mass.), Blackwell Publishers, 1996, G 9-7102

FAVENNEC, CHEVALIER, AUGER ... Géopolitique de l'énergie: besoins, ressources, échanges mondiaux, Paris, Éd. Technip, 2009, G 9-7154

FERRATON, TOUZARD, CHALLEMEL DU ROZIER ... Comprendre l'agriculture familiale: diagnostic des systèmes de production, Versailles, Gembloux, Quae, CTA, Presses agronomiques de Gembloux, 2009, G 9-7158 + CD 345

Francophonie et relations internationales, Paris, Agence universitaire de la Francophonie, Ed. des Archives contemporaines, 2009, G 9-7179

FUMEY, Les champs du monde : nourrir la planète, Grenoble, Glénat, 2009, AQ 9-71

FUMEY, ETCHEVERRIA, PITTE ... Atlas mondial des cuisines et gastronomies; supplément Cultures alimentaires et mondialisation, Paris, Autrement, 2009, G 9-7177

FUMEY, VARLET, ZEMBRI, Mobilités contemporaines: approches géoculturelles des transports, Paris, Ellipses, 2009, G 9-7186

FUSCO GIRARD, NIJKAMP, Cultural tourism and sustainable local development, Farnham, Burlington (VT), Ashgate, 2009, G 9-7084

GAZIER, La crise de 1929, Paris, Presses universitaires de France, 2009, K 1075

GEMENNE, Géopolitique du changement climatique, Paris, A. Colin, 2009, G 9-7187

GENTELLE, Géopolitique du monde contemporain : états, continents, puissances, Paris, Nathan, 2008, G 9-7117

GILBERT, GILBERT, La distance, objet géographique, Rennes, Cercle de réflexion universitaire du Lycée Chateaubriand, 2009, G 9-7091

GRANDJEAN, Construction identitaire et espace, Paris, L'Harmattan, 2009, G 9-7176

GUILLAUME, Vaincre la faim : pour en finir avec l'inacceptable, Paris, Eyrolles, 2009,

G 9-7171

GUILLAUMET, LAQUES, LÉNA, La spatialisation de la biodiversité : pour la gestion durable des territoires, Marseille, IRD, 2009, G 9-7175

GRESH, L'Atlas 2010, Paris, A. Colin ; Le Monde diplomatique, 2009, G 9-7080

GUNNELL, Écologie et société: repères pour comprendre les questions d'environnement, Paris, A. Colin, 2009, G 9-7120

Habiter : [exposition, Grenoble, Musée dauphinois, 26 avril 2009-30 juin 2010], Grenoble, Musée dauphinois, Isère, Conseil général, 2009, G 9-5962

HALDRUP, LARSEN,Tourism, performance and the everyday : consuming the Orient, London, Routledge, 2010, G 9-7183

HALL, Markusen, Silicon landscapes, Boston, Allen and Unwin, 1985, G 9-7170

HALL, WILLIAMS,Tourism and innovation, London, Routledge, 2008, G 9-7195

HERVÉ, LALOË, Modélisation de l'environnement: entre natures et sociétés, Versailles, Nanterre, Éd. Quæ ; NSS-Dialogues, 2009, G 9-7064

HESSE, Markus, The city as a terminal : the urban context of logistics and freight transport, Aldershot, Burlington (Vt.), Ashgate, 2008, G 9-7088 
Infectious diseases : a geographical analysis : emergence and re-emergence, Oxford, Oxford university press, 2009, G 9-7078

JAFFRELOT, LEQUESNE, L'enjeu mondial: les migrations, Paris, Presses de Sciences Po, L'Express, 2009, G 9-7170

LACOSTE, Atlas géopolitique, Paris, Larousse, 2007, G 9-7119

LARSEN, Jonas, Mobilities, networks, geographies, Aldershot, Burlington (Vt.), Ashgate, 2006, G 9-7090

LASSERRE, ROCARD, Écologie, irrigation, diplomatie, comment éviter les guerres de l'eau : l'eau au coeur des conflits du XXIe siècle, Paris, Delavilla, 2009, G 9-7156

LE BRAS, Vie et mort de la population mondiale, Paris, Le Pommier, Cité des sciences et de l'industrie, 2009, G 9-7159

LECOURS, NOOTENS, Dominant nationalism, dominant ethnicity : identity, federalism and democracy, Bruxelles, PIE P. Lang, 2009, G 9-7198

LE GUIRRIEC-MILNER, L'essentiel des mécanismes de l'économie, Paris, Gualino-Lextenso, 2009, G 9-7150

LEWIS, KANJI,Non-governmental organizations and development, London, New York, Routledge, 2009, G 9-7182

LA SOUDIÈRE, TABEAUD, Météo du climat et des hommes, Paris, Presses universitaires de France, 2009, G 9-7130

LESLIE, Tourism enterprises and sustainable development : international perspectives on responses to the sustainability agenda, New York, Routledge, 2009, G 9-7086

LIOGIER, LEGEAY, La mondialisation du champ religieux, nouveaux acteurs et nouveaux enjeux, Bruxelles, 2009, G 9-7070

LOCHE, TALLAND, Quand les quartiers réinventent la ville: les acteurs de la rénovation urbaine, Paris, Autrement, 2009, G 9-7141

MACCAGLIA, MATARD-BONUCCI, NICOLAS, Atlas des mafias : acteurs, trafics et marchés de la criminalité organisée, Paris, Éditions Autrement, 2009, G 9-7184

MERLIN, L'urbanisme, Paris, Presses universitaires de France, 2009, K 1073

MOLSTAD, BESANÇON, Où partir avant qu'il ne soit trop tard: compte à rebours pour un tourisme responsable, Paris, La Découverte, 2009, G 9-7166

MC DERMOTT, TAYLOR,Industrial organization and location, Cambridge, London, NewYork, Cambridge university press, 1982, G 9-7128

MENDELSOHN, Robert O., Climate change and agriculture : an economic analysis of global impacts, adaptation and distributional effects, Cheltenham, Northampton, E. Elgar, 2009, G 9-7074

MÉNDEZ GUTIÉRREZ DEL VALLE, Ricardo, Geografía económica : la lógica espacial del capitalismo global, Barcelona, Ed. Ariel, 1997, G 9-7113

MONNET, STASZAK, Le consommateur ambulant, Toulouse, Erès, 2009, G 9-7116

MOREAU DEFARGES, MONTBRIAL, Ramses 2010: rapport annuel mondial sur le système économique et les stratégies, Paris, Dunod, Institut français des relations internationales, 2009, G 9-7122 
MOUHOUD, Mondialisation et régionalisation des économies, Noisy-le-Grand, Institut de recherches économiques et sociales, 1998, G 9-7104

MVOGO, Thomas Noah, Mondialisation et sous-développement : la réalité des pays pauvres, Paris, L'Harmattan, 2009, G 9-7082

ORIGET DU CLUZEAU, TOBELEM, KNAFOU, Culture, tourisme et développement: les voies d'un rapprochement, Paris, l'Harmattan, 2009, G 9-7149

PAULET, Jean-Pierre, Géographie urbaine, Paris, A. Colin, 2009, G 9-7058

PAULET, Jean-Pierre, Manuel de géographie urbaine, Paris, A. Colin, 2009, G 9-7059

PITTOCK, Barrie A., Climate change : the science, impacts and solutions, London, Collingwood, VIC ; Earthscan, CSIRO, 2009, G 9-7073

POLÈSE, SHEARMUR, Économie urbaine et régionale: introduction à la géographie économique, Paris, Economica, 2009, G 9-7153

REICH, Robert B., L'économie mondialisée, Paris, Dunod, 1993, G 9-7106

REMACLE, WINAND, America, Europe, Africa : 1945-1973, Bruxelles, PIE - P. Lang, 2009, G 9-7152

ROQUES, CHIGNIER-RIBOULON, La fin des petites villes : une modernité envahissante, Paris, L'Harmattan, 2009, G 9-7173

ROSE, Adam, The economics of climate change policy, international, national and regional mitigation strategies, Northampton (Mass.), Cheltenham, Edward Elgar, 2009, G 9-7076

RUTH, IBARRARÁN, Distributional impacts of climate change and disasters : concepts and cases, Cheltenham (UK), Northampton, (MA) E. Elgar, 2009, G 9-7077

SANTANDER, L'émergence de nouvelles puissances : vers un système multipolaire ?: Afrique du Sud, Brésil, Chine, Inde, Mexique, Russie, Paris, Ellipses, 2009, G 9-7062

SAUVAIN-DUGERDIL, THIRIAT, Développer le genre en démographie : de la naissance à l'âge adulte, Paris, Centre Population et développement, 2009, G 9-7139

SECCHI, GRILLET-AUBERT, La ville du vingtième siècle, Paris, Éd. Recherches, 2009, G 9-7160

SCHOLTEN, VELDE, MANEN,Geospatial technology and the role of location in science, Dordrecht, Heidelberg, London, Springer, 2009, G 9-7135

SHORT, John Rennie, The urban order : an introduction to cities, culture and power, Cambridge (Mass.), Blackwell, 1996, G 9-7110

SHOVAL, ISAACSON,Tourist mobility and advanced tracking technologies, Abingdon (UK), New York, Routledge, 2010, G 9-7161

SMITH,The state of the world atlas, London, Earthscan, 2008, G 9-7181

SPINDLER, HURON, L'évaluation de l'événementiel touristique, Paris, L'Harmattan, 2009, G 9-7189

STÉBÉ, MARCHAL, Traité sur la ville, Paris, Presses universitaires de France, 2009, G 9-7071

URRY, John, The tourist gaze : leisure and travel in contemporary societies, London, Sage, 1990, G 9-7105

VAINOPOULOS, MERCIER, Le tourisme, Paris, le Cavalier bleu, 2009, G 9-7144

VALLEGA, Fondamenti di geosemiotica, Roma, Società geografica italiana, 2008, G 9-7197 

2009, G 9-7121 8-3244 2009, G 9-7196 8-3251

\section{Mélanges}

\section{Monde arabe} G 24-439

VARET, 10 enjeux des géosciences: dossier spécial année internationale de la planète Terre, Orléans, BRGM, 2009, G 9-7134

VELASCO-GRACIET, Territoires, mobilités et sociétés: contradictions géographiques et enjeux pour la géographie, Pessac, Maison des sciences de l'homme d'Aquitaine, 2009, G 9-7146 WIHTOL DE WENDEN, La globalisation humaine, Paris, Presses universitaires de France,

\section{Géographie physique}

BRAVARD, Jean-Paul, La géologie: les sciences de la Terre appliquées à l'archéologie, Paris, Ed. Errance, 2009, G 8-3247

COURTILLOT, Nouveau voyage au centre de la Terre, Paris, O. Jacob, 2009, G 8-3248

FOUCAULT, Climatologie et paléoclimatologie, Paris, Dunod, 2009, G 8-3249

GODARD, Alain, Les climats : mécanismes, variabilité, répartition, Paris, A. Colin, 2009, G

LAGANIER, ARNAUD-FASSETTA, Les géographies de l'eau : processus, dynamique et gestion de l'hydrosystème, Paris, L'Harmattan, 2009, G 8-3252

LATTEUX, Bernard, Exploitation de matériaux marins et stabilité du littoral: exposé des mécanismes, recommandations sur les études à mener, Versailles, Quae, 2008, G 8-3243

LE TREUT, Nouveau climat sur la terre: comprendre, prédire, réagir, Paris, Flammarion,

RUDDIMAN, PIETRASIK, La charrue, la peste et le climat, Courbevoie, Randall, 2009, G

WESTBROEK, JEANMOUGIN, Terre! : des menaces globales à l'espoir planétaire, Paris, Ed. du Seuil, 2009, G 8-3250

[Hommage à Orlando Ribeiro], Lisboa, Centro de estudos geográficos, 2008, G 6-344

DAMIEN, MARCADON, Les ports européens et la mondialisation : la réforme française, Paris, l'Harmattan, 2009, G 6-344

BOUGANIM, MILROD, Tel-Aviv sans répit, Paris, Autrement, 2009, G 24-437

DONATI, L'exception syrienne : entre modernisation et résistance, Paris, la Découverte, 2009,

ENCEL, Géopolitique du sionisme : stratégies d'Israël, Paris, A. Colin, 2009, G 24-435

FATTÉ DAVIE, Fonctions, pratiques et figures des espaces publics au Liban: perspectives comparatives dans l'aire méditerranéenne, Beyrouth, Tours, Académie libanaise des BeauxArts ; Centre d'histoire de la ville moderne et contemporaine, 2007, G 24-434

2 Gens de Marrakech: géo-démographie de la ville Rouge, Paris, Institut national d'études démographiques, 2009, G 24-436 
Le Golfe et ses émirats, Paris, La Découverte, 2009, G 24-432

MARRINER, Géoarchéologie des ports antiques du Liban, Paris, l'Harmattan, 2009, G 24-438

MEYNADIER, Pascal, L'Égypte, au cœur du monde arabe: l'heure des choix, Perpignan, Tempora ; éd. du Jubilé, 2009, G 24-433

MIKAÏL, Barah, La Syrie en cinquante mots clés, Paris, l'Harmattan, 2009, G 24-431

Tunisia's global integration : a second generation of reforms to boost growth and employment, Washington, The World Bank, 2009, G 24-440

\section{Périodiques}

ALPHANDÉRY, BILLAUD, La sociologie rurale en questions, Paris, Éditions de l'École des hautes études en sciences sociales, 2009, P 1511-183

BATAILLON, Amérique centrale, fragilité des démocraties, Paris, Choiseul, 2009, P 4389-73

BERGER, POUSIN, Espaces du quotidien, Paris, CNRS, 2008, P 4105-14

BETHEMONT, Les grands projets hydrauliques et leurs dérives, Lyon, Revue de géographie de Lyon, 2009, P 487-84-1/2

BRAS, La fabrique de la mémoire : variations maghrébines : dossier, Paris, CNRS éd., 2008, P 4719-2008

Crise mondiale, crise sociale: les territoires dans la tourmente, Paris, A. Colin, 2009, P 1354-2010

DESVIGNES, Qualité \& tourisme: du marketing au management, Paris, Editions touristiques européennes, 2009, P 4198-102

DESVIGNES, LE CHUITON, Nuit urbaine \& tourisme, Paris, Editions touristiques européennes, 2009, P 4198-103

DUPUY, LAVIGNE, MORIN, Géographies de la finance mondialisée, Paris, la Documentation française, 2009, P 797-5299

Eau, pêche, tourisme rural, conflits d'usage: varia, Rennes, Presses universitaires de Rennes, 2009, P 1167-211

Entrechocs culturels : espaces et tensions sociétales, Paris, L'Harmattan, 2009, P 4350-68

ERKENS, Sediment dynamics in the Rhine Catchment : quantification of fluvial response to climate change and human impact, Utrecht, Koninklijk Nedelands Aardrijkskundig Genootschap, 2009, P 3619/B-388

Géopolitique de l'Afrique subsaharienne, Paris, Institut international d'études stratégiques, 2009, P 4610-25

La géostratégie de la Russie, Paris, Institut international d'études stratégiques, 2009, P 4610-24

L' impasse au Tibet, Hong-Kong, CEFC, 2009, P 4681-108-3

Islands, research and concepts : dossier, Paris, Insula, 2009, P 4414-18-1

JARNOUX, Philippe, Terres de conquête, terres en déprise: enjeux fonciers, agricoles et cynégétiques, Caen, Maison de la Recherche en sciences humaines de Caen, 2009, P 4515-12 

4532-199 P 797-5303 3074-54/55 797-5300/01

LABROUSSE, BATAILLON, L'Uruguay gouverné à gauche, une voie singulière en Amérique latine?, Paris, Choiseul, 2009, P 4389-74

PAVLOPOULOS, Palaeo-environmental dynamics and archaeological sites, Berlin, Gebrüder Borntraeger, 2009, P 10 BIS-53-1

MA MUNG, Nouvelles migrations chinoises en Afrique et en Amérique latine, Poitiers, Association pour l'étude des migrations internationales, 2009, P 4045-25-1

Les migrations au Proche-Orient, Paris, Choiseul, La Documentation française, 2009, P

MERLIN, L'exode urbain : de la ville à la campagne, Paris, La Documentation française, 2009,

Milieux naturels et paléomilieux des Vosges au bassin de la Sarre, Nancy, Association de Géographes de l'Est, Université de Nancy 2, 2009, P 1422-47-4

NIJS,Modelling land use change : improving the prediction of future land use patterns, Utrecht, Koninklijk Nederlands Aardrijkskundig Genootschap, 2009, P 3619/B-386

Pillages et pirateries, Paris, La Découverte, 2009, P 3536-134

La puissance militaire chinoise, Paris, Choiseul, 2009, P 4741-18

PRÉVOT, Les agences d'urbanisme en France: l'expérimentation, l'innovation et leurs limites, Lille, Université des sciences et technologies de Lille, UFR de Géographie et d'Aménagement, 2009, P 1632/A-2007-2

RAMOUSSE, SALIN, La question environnementale en Amérique latine, Paris, IHEAL, 2009, P

RÉMOND, Les retraites en question, Paris, La Documentation française, 2009, P

SCHNEIDER-SLIWA, BHATT,Recovering slums : determinants of poverty and upward social mobility in urban slums, case studies from India, Basel, University of Basel, Department of Geography, 2008, P 4788-1

SIBERTIN-BLANC, Cultures et projets de territoire, Toulouse, Presses universitaires du Mirail, 2009, P 460/A-27

Terrassement, Paris, LCPC, 2009, P 4276-274

Tibet : special issue, New York, The American Geographical Society, 2009, P 1079/A-52-2

Variations, Bondy, IRD, 2009, P 1652/A-51

Vers une société harmonieuse, Paris, Choiseul, 2009, P 4741-19

VISSEREN-HAMAKERS,Partnerships in biodiversity governancean assessment of their contributions to halting biodiversity loss, Groningen, Koninklijk Nederlands Aardrijkskundig Genootschap, 2009, P 3619/B-387

VOLVEY, Spatialités de l'art, Reims, ERIGUR, 2009, P 3117-129/130

VÖTT, BRÜCKNER, Ergebnisse aktueller KüstenforschungBeiträge der 26. Jahrestagung des Arbeitskreises "Geographie der Meere und Küsten"25.-27. April 2008 in Marburg, Marburg, Marburger Geographische Gesellschaft, 2009, P 1319-145

WAACK,Randerscheinungen : regionalisierungen und skalierungen im Kontext von Transformations- und Globalisierungseffekten in der Kontroverse um den Goldbergbau im rumänischen Westgebierge, Leipzig, Leibniz-Institut für Länderkunde, 2009, P 12-63 


$$
\text { P 4532-200 }
$$

\section{Thèses} 2061 2007, T 2056 2002, T 2063

BARON, Dossier pour l'obtention du diplôme d'habilitation à diriger des recherches, 2000, T

CHAPILLON, AILLAUD, Les collectivités locales face à l'aménagement numérique de leur territoire: quelles solutions pour le déploiement des réseaux de communications électroniques à très haut débit? : exemple du SIPPEREC et de Saint-Quentin-en-Yvelines, 2008, D 2733

CLERVAL, Anne, La gentrification à Paris intra-muros: dynamiques spatiales, rapports sociaux et politiques publiques, S.l., s.n., 2008, T 2055

FLEURY, Antoine, Les espaces publics dans les politiques métropolitaines: réflexions au croisement de trois expériences : de Paris aux quartiers centraux de Berlin et Istanbul, S.l., s.n.,

GÉNEAU DE LAMARLIÈRE, Des nations de l'Europe aux régions européennes: les nouveaux espaces de la production agricole : maîtrise de géographie et d'aménagement, D 2735

JANIN, L'immuable, le changeant et l'imprévu : les économies de plantation bamiléké et béti du Cameroun confrontées aux chocs extérieurs, Paris, 1995, T 2064

5 LESTRADE, Images, stratégies et pratiques des centres commerciaux dans la recomposition des espaces marchands et socioculturels des banlieues parisiennes: thèse pour l'obtention du doctorat, 1999, T 2066

MALEVAL, Le Lac de Saint-Pardoux et l'évolution morphologique des lacs de barrage artificiel,

MARESCOT DU THILLEUL, L'influence de la peinture sur le paysage du jardin : le jardin public parisien : mémoire présenté en vue de l'obtention de la maîtrise de géographie, 2000, T 2736

MARTOUZET, Normes et valeurs en aménagement-urbanisme: limites de la rationalité et nécessité de prise en compte du multi-niveaux, 2002, T 2067

MARMIGNON, Paysage et socialité à Ōsaka depuis Meijila création de l'urbain et du périurbain au Japon : vers une nouvelle socialité ?, Paris, 2008, T 2065

SANJUAN, Les projets d'aménagements urbains à Shanghai: l'Est de l'agglomération shanghaïenne (Pudong, les arrondissements de Hongkou et de Yangpu), 1989, D 2734

1 VENIER, Travail dans le Golfe Persique et développement au Kérala: les migrants internationaux, des acteurs au coeur des enjeux sociaux et territoriaux ?, 2003, T 2062 